REVISTA DE DERECHO UNED, NÚM. 17, 2015

\title{
LA DIVERSIDAD CULTURAL COMO DERECHO FUNDAMENTAL DE LOS PUEBLOS INDÍGENAS
}

\author{
CULTURAL DIVERSITY AS A FUNDAMENTAL RIGHT \\ OF INDIGENOUS PEOPLES
}

\author{
CAYETANo NúÑEz Rivero \\ (UNED). España \\ Carla Z. PÉrez A
}

(URG). Venezuela. Centro de Investigaciones Antropológicas de la Universidad Nacional Experimental de Guayana

Resumen: En este ensayo analizamos la diversidad cultural en Venezuela y su desarrollo como derecho fundamental para los pueblos indígenas. El reconocimiento en la Constitución de 1999 de Venezuela como una sociedad multiétnica y pluricultural constituye un avance significativo, dada la importancia y las características de los pueblos indígenas para quienes es necesario crear garantías que permitan su desarrollo como pueblos con especificidad cultural, pues cada pueblo indígena posee una identidad, lengua, costumbres, historia y unos valores propios, que se ejercen como el derecho humano a la cultura. La multiculturalidad implica el reconocimiento de varias culturas en un mismo territorio, por lo tanto el derecho a la cultura debe ser concebido como derecho que permite ejercer libertades individuales que determina un derecho de la colectividad a constituirse por si misma en convivencia con las otras. Por ello, la imposición de figuras externas a la propia organización indígena como los consejos comunales en el caso venezolano, vulneran el derecho a la cultura propia, situación que los pone en desventaja. Por ello es necesario que los pueblos indígenas gocen del derecho a la libre determinación que les permita desarrollar su condición política, económica, social y cultural. 
Palabras clave: Diversidad Cultural, Derechos Humanos, Cultura, Pueblos Indígenas.

Abstract: The importance of this documentary research, lies in the analysis of cultural diversity in Venezuela and its development as a fundamental right for indigenous peoples. For the Venezuelan State constitutes a significant advance in the 1999 Constitution, his renown as a multiethnic and multicultural society, given the importance and characteristics of indigenous peoples is necessary to create guarantees for their development as peoples with cultural specificity, as each indigenous community has an identity, language, customs, history and values, this translates into the human right to culture. Multiculturalism implies recognition of various cultures in the same territory, therefore the right to culture should be conceived as rights entitling to exercise individual liberties which determines a right of the public to constitute itself. However, in the case of Venezuela's indigenous peoples externally imposed the indigenous organization itself as the communal councils, among others, figures violate the right to their own culture, a situation that puts them at a disadvantage, so it is necessary that indigenous peoples have the right to self-determination enabling them to develop their political, economic, social and cultural status.

Keywords: Cultural Diversity, Human Rights, Culture, Indigenous Peoples.

Recepción original: 27/08/2015

Aceptación original: 30/09/2015

Sumario: 1. Introducción. 2. El Derecho a la Cultura. 3. Multiculturalidad, Interculturalidad y Pueblos Indígenas. 4. Conclusión. 5. Referencias.

Summary: 1. Introduction. 2. The Right to Culture. 3. Multiculturalidad, Intercultural and Indigenous Peoples. 4. Conclusion. 5. Referencias.

\section{INTRODUCCIÓN}

La cultura, engloba todos los componentes que conforman una sociedad, que van desde las creencias, convicciones, idiomas, saberes, entre otros aspectos, que corresponden a la existencia y desarrollo de la persona humana.

La cultura establece el eje fundamental en el que se realiza el hombre, determina sus rasgos distintivos y permite su desarrollo den- 
tro de una sociedad o grupo social, generando la necesidad de su trascendencia para el mantenimiento de esa cultura.

El ejercicio de la cultura constituye un derecho fundamental, garantizado y protegido por los distintos pactos y convenios internacionales; y en el caso de los pueblos indígenas la garantía del desarrollo de su cultura está íntimamente relacionada con sus territorios y el derecho a la libre determinación de estos pueblos, como un medio de protección a su cosmovisión cultural y formas de vida.

La finalidad de este estudio fue analizar la diversidad cultural en Venezuela y su desarrollo como derecho fundamental para los pueblos indígenas, visto que el Estado venezolano, se erige como un Estado con una sociedad multiétnica y pluricultural.

La presente investigación se lleva a cabo dentro del diseño documental, y se estructuró en dos apartados, en un primer apartado se encuentra el derecho a la cultura y el segundo y último apartado se encuentra la multiculturalidad, interculturalidad y los pueblos indígenas.

\section{EL DERECHO A LA CULTURA}

La cultura es concebida como un elemento esencial para el desarrollo de la vida en sociedad, ésta incluye el conjunto de formas, o modelos, explícitos o no, por medio de los cuales una sociedad codifica el comportamiento de las personas que la conforman; sus costumbres, creencias, religión, códigos de conducta ${ }^{1}$, entre otros aspectos que desarrolla el individuo que vive en comunidad.

La Declaración de la UNESCO sobre las Políticas Culturales de $1982^{2}$, establece que:

(...) en su sentido más amplio, la cultura puede considerarse actualmente como el conjunto de los rasgos distintivos, espirituales y materiales, intelectuales y afectivos que caracterizan a una sociedad o un grupo social. Ella engloba, además de las artes y las letras, los modos de vida, los derechos fundamentales al ser humano, los sistemas de valores, las tradiciones y las creencias, y que la cultura da al hombre la capacidad de reflexionar sobre sí mismo. Es ella la que hace de nosotros seres especifi-

${ }^{1}$ GOIG, Juan (2013). Inmigración y Diversidad Cultural. Sobre los «Derechos Culturales de los Inmigrantes», en Sobre los "Derechos Culturales de los Inmigrantes», en Libro Colectivo Derechos Humanos: Problemas Actuales (Fundación Cultural Enrique Luño- Peña), Universitas S. A., vol. II, Madrid, págs. 1317-1339.

2 Declaración de México sobre las Políticas Culturales. Conferencia mundial sobre las políticas culturales México D. F., 26 de julio - 6 de agosto de 1982. 
camente humanos, racionales, críticos y éticamente comprometidos. A través de ella discernimos los valores y efectuamos opciones. A través de ella el hombre se expresa, toma conciencia de sí mismo, se reconoce como un proyecto inacabado, pone en cuestión sus propias realizaciones, busca incansablemente nuevas significaciones, y crea obras que lo trascienden.

Asimismo la Declaración de Friburgo (2003), establece que:

El término "cultura» abarca los valores, las creencias, las convicciones, los idiomas, los saberes y las artes, las tradiciones, instituciones y modos de vida por medio de los cuales una persona o un grupo expresa su humanidad y los significados que da a su existencia y a su desarrollo ${ }^{3}$.

El derecho a la cultura constituye un derecho humano reconocido en el ámbito jurídico internacional, así la Declaración Universal de los Derechos Humanos en su artículo 27 señala: «Toda persona tiene derecho a tomar parte libremente en la vida cultural de la comunidad (...)» ${ }^{4}$. Este derecho tiene dimensiones dentro de la identidad del ser humano, por ser estas características culturales y tradicionales practicadas en común con otros individuos al pasar el tiempo ${ }^{5}$, por ello, la importancia de las culturas, no por sí mismas, sino porque solo a través del acceso a la cultura, estos obtienen el acceso a una serie de opciones determinantes para su vida ${ }^{6}$.

El derecho a la cultura propia, determina el elemento primario dentro del marco constitucional, por lo que se considera un derecho esencial para cualquier individuo. Este es un derecho constituyente, no uno más, sino el que determina la comunidad primaria, es el ámbito colectivo primario donde se desarrolla el individuo, por lo que se constituye en el primer espacio de orden jurídico y político, que al reconocer y respetar libertades se denomina constitucional, debido a que la significación cultural genera la participación en lengua y costumbres, en comportamientos individuales y respuestas sociales, en asunciones y expectativas conjuntas de donde se compone la sociedad ${ }^{7}$.

${ }^{3}$ Declaración de Friburgo sobre los Derechos Culturales, del 7 de mayo de 2007, artículo 2.

${ }^{4}$ Declaración Universal de los Derechos del Hombre, Adoptada y Aprobada por la Asamblea General de las Naciones Unidas, del 10 de diciembre de 1948.

${ }^{5}$ GOIG, Juan (2013). Ob. cit., pág. 1320.

${ }^{6}$ DWORKIN, Ronald (1985). A Matter of Principle. Cambridge, Harvard University Press, págs. 232-233.

7 CLAVERO, Bartolomé (2004). Multiculturalismo Constitucional con perdón, de veras y en frío, en Avances en la protección de los derechos de los pueblos indígenas, 
Es el derecho a una cultura determinada, en la que los seres humanos se hagan capaces de libertades, lo que permite que los individuos se constituyan como tales, es decir, el derecho a la cultura debe ser concebido como derecho que permite ejercer libertades individuales que determina un derecho de la colectividad a constituirse por sí misma ${ }^{8}$, es por ello que, el derecho a la cultura se le considera un derecho doble por abarcar derechos individuales y a su vez derechos colectivos $^{9}$, que para su garantía requiere de la realización y del ejercicio efectivo de todos los derechos humanos.

Conforme a las obligaciones que establece la Declaración Universal de los Derechos Humanos, la cual señala en su artículo 29 que «toda persona tiene deberes respecto a la comunidad puesto que solo en ella puede desarrollar libre y plenamente su personalidad $»^{10}$, se pudiera afirmar entonces, que por ser dentro de la comunidad donde adquiere sentido el desarrollo en libertad y en pleno de la personalidad del individuo, ésta le da derecho a participar en la vida cultural de su comunidad, por ser la cultura la que le permite identificarse plenamente como miembro de dicha comunidad, generando así su identidad cultural.

La identidad cultural, por su parte, «debe entenderse como el conjunto de referencias culturales por el cual una persona, individual o colectivamente, se define, se constituye, comunica y entiende ser reconocida en su dignidad ${ }^{11}$, es decir, constituye todo el contexto de referencias culturales por las que una persona o un grupo social se define, se manifiesta y desea ser reconocido ${ }^{12}$.

Para los pueblos indígenas, el derecho a la propia cultura y su identidad cultural comprende la protección a sus expresiones artísticas, arquitectónicas, cosmovisión religiosa, costumbres y tradiciones ancestrales ${ }^{13}$, lugares sagrados y de culto. No obstante, a los pueblos

(Fernando Mariño y J. Daniel Oliva, editores), Instituto Universitario de Estudios Internacionales y Europeos «Francisco de Vitoria. Editorial Dykinson, Madrid, pág. 159.

${ }^{8}$ CLAVERO, Bartolomé (2004). Ob. cit., pág. 158.

9 Ibidem.

10 Declaración Universal de los Derechos del Hombre, Adoptada y Aprobada por la Asamblea General de las Naciones Unidas, del 10 de diciembre de 1948.

${ }_{11}$ Declaración de Friburgo sobre los Derechos Culturales del 7 de mayo de 2007, artículo 2.

12 RUIZ, Oswaldo (2004). El derecho a la identidad cultural de los pueblos indígenas y la minorías nacionales: Una mirada desde el sistema interamericano, en SUR - Revista Internacional de Derechos Humanos, No. 5, Año 3, pág. 45.

13 COLMENARES, Ricardo (2001). Los derechos de los pueblos indígenas, Editorial Jurídica Venezolana, Caracas, pág. 4. 
indígenas se les ha considerado como «atrasados» por algunos gobiernos, y han sido víctimas de políticas agresivas de asimilación cultural; en muchas ocasiones sus conocimientos y artes, han sido destruidos y no se consideraban tesoros dignos de ser mantenidos. Si bien, existen algunos países que han promulgado leyes para determinar los lugares de importancia histórica y cultural, no siempre se han aplicado de manera coherente, en especial lo que respecta, por ejemplo, a la protección y el respeto de los lugares sagrados de los pueblos indígenas, igualmente estos gobiernos no han evitado disponer de esos lugares o desarrollarlos con otros fines ${ }^{14}$.

Es por ello, que el acervo cultural indígena amerita protección, debido a que el dominio y control de sus territorios por parte de los Estados, pone en peligro su conocimiento tradicional, su patrimonio y por ende su existencia.

La protección de la cultura tradicional y popular, se garantiza debido a la fragilidad extrema de algunas formas de esta cultura como la de los pueblos indígenas $\mathrm{y}$, en especial la que corresponde a las tradiciones orales, y el peligro de que estas se pierdan; para ello fue aprobada por la UNESCO, la Recomendación sobre la Salvaguardia de la Cultura Tradicional y Popular. En esta recomendación se define a la cultura tradicional y popular, como creaciones culturales que corresponden a la expresión de su identidad cultural, que se transmiten oralmente, comprende la lengua, la música, entre otras artes. Asimismo en el Preámbulo de la mencionada Recomendación se afirma que la cultura tradicional o popular «forma parte del patrimonio universal de la humanidad y que es un poderoso medio de acercamiento entre los pueblos y grupos sociales existentes y de afirmación de su identidad cultural» ${ }^{15}$.

La conservación de la cultura y de la identidad étnica de los pueblos indígenas se ha visto amenazada por la aculturación que inició producto de las misiones evangelizadoras, y también por la discriminación que está presente en muchas regiones del mundo y estos pueblos indígenas no escapan a ella, pues se les ha privado de sus derechos humanos y libertades fundamentales principalmente porque les han sido arrebatadas sus tierras y sus recursos, ya sea por los colonizadores o por el Estado donde habitan.

14 DAES, Erica (1997). Protección del patrimonio de los pueblos Indígenas, Informe de la Subcomisión de Prevención de Discriminaciones y Protección a las Minorías, Relatora-Presidenta del Grupo de Trabajo sobre Poblaciones Indígenas, pág. 3.

15 Ibidem. 
Producto de la evidente violación de los derechos civiles y políticos que los pueblos indígenas han padecido, a lo largo de la historia, por la actitud discriminatoria por parte de la sociedad en general, que había generado que se considerara que «los indígenas son inferiores que el resto de los ciudadanos, por lo tanto no tienen derechos» ${ }^{16}$, el «Comité para la eliminación de la discriminación racial», de la ONU, en su Recomendación general 23, ha exhortado a los Estados parte, para que reconozcan y respeten la cultura, historia, idioma y formas de vida de las poblaciones indígenas como factor de enriquecimiento de la identidad cultural del Estado y garanticen su preservación. Deben para ello, proporcionar a estas poblaciones las condiciones necesarias que les permita el desarrollo económico y social sostenible, compatible con sus características culturales, igualmente la garantía de que las comunidades indígenas puedan ejercer su derecho a practicar y reavivar sus tradiciones y costumbres culturales y a preservar y practicar su idioma.

Por su parte, el Pacto Internacional de Derechos Civiles y Políticos en su artículo 27, dispone que:

En los Estados en que existan minorías étnicas, religiosas o lingüísticas, no se negará a las personas que pertenezcan a dichas minorías el derecho que les corresponde, en común con los demás miembros de su grupo, a tener su propia vida cultural, a profesar y practicar su propia religión y a emplear su propio idioma ${ }^{17}$.

$\mathrm{Su}$ importancia radica en el derecho que tienen las personas pertenecientes a un grupo o minoría, al disfrute y ejercicio de su cultura determinada, que guarda relación con los modos de vida vinculados estrechamente al territorio y al uso de los recursos, que corresponde a los miembros de comunidades indígenas que pueden constituir una minoría. Aunque estos derechos reconocidos, en el articulo señalado, corresponden a derechos individuales, estos dependen a su vez de la capacidad del grupo para conservar su cultura, su idioma o su religión, por lo que la protección de estos derechos tiende a garantizar la preservación y el desarrollo continuado de la identidad cultural, religiosa, y social de las minorías interesadas, enriqueciendo su tejido social. En consecuencia estos derechos deben ser protegidos como tales, sin que se les confunda con otros derechos personales otorgados a todas y cada una de las personas ${ }^{18}$.

16 COLMENARES, Ricardo (2001). Ob. cit., pág. 71.

17 Pacto Internacional de Derechos Civiles y Políticos, del 23 de marzo de 1976.

18 Comité de Derechos Humanos (1994). Observaciones Generales de los Comités sobre los pueblos indígenas, Observación General No. 23, Los derechos de las Minorías étnicas, religiosas o lingüística, artículo 27 del Pacto. 
Igual reconocimiento a la protección de los derechos culturales e intelectuales de los pueblos indígenas hace la Declaración de Mataatua, sobre los Derechos de Propiedad Cultural e Intelectual de los Pueblos Indígenas ${ }^{19}$, que establece que estos son dueños exclusivos de su propiedad cultural e intelectual, garantizado por la autodeterminación y asociado con el territorio y al control de sus recursos.

En este sentido, la protección de la propiedad cultural e intelectual, está vinculada esencialmente a la realización de los derechos territoriales y de la libre determinación de los pueblos indígenas. Sus conocimientos tradicionales relativos a valores, autonomía o autogobierno, organización social, gestión de los ecosistemas, mantenimiento de la armonía entre los pueblos y respeto de la tierra están enraizados en las artes, las canciones, la poesía y la literatura que cada generación de niños indígenas debe aprender y renovar. Estas variadas y ricas expresiones de la identidad específica de cada pueblo indígena aportan la información necesaria para mantener, desarrollar y, de ser necesario, restablecer las sociedades indígenas en todos sus $\operatorname{aspectos}^{20}$.

\section{MULTICULTURALIDAD, INTERCULTURALIDAD Y PUEBLOS INDÍGENAS}

Los avances dentro de las sociedades las han transformado en sociedades en las que conviven posturas diferenciadas en lo cultural y en lo político, y entre las cuales, la convivencia no está ajena al levantamiento de conflictos. Esta diversidad cultural no se erige como un modelo normativo planificado de cómo debe ser la sociedad, sino que es un hecho que se impone, y que implica la coexistencia de grupos con códigos de conducta que se oponen radicalmente, y entre los que pueden surgir puntos de fricción ${ }^{21}$.

La multiculturalidad implica el reconocimiento de varias culturas en un mismo territorio, esta hace referencia a la existencia de varias culturas diferentes, sin embargo esta no va más allá, es decir, que en

${ }^{19}$ Declaración de Mataatua, sobre los Derechos de Propiedad Cultural e Intelectual de los Pueblos Indígenas, junio de 1993.

${ }^{20}$ DAES, Erica (1997). Protección del patrimonio de los pueblos Indígenas, Informe de la Subcomisión de Prevención de Discriminaciones y Protección a las Minorías, Relatora-Presidenta del Grupo de Trabajo sobre Poblaciones Indígenas, pág. 16.

${ }^{21}$ MARTÍNEZ DE PISÓN CAVERO, J. (2003). Ciudadanía e inmigración en las sociedades multiculturales, en Ciudadanía e inmigración (Pomed Sanchez, I. y Velasco Caballero, F. Editores), Monografías de la Revista Aragonesa de Administración Pública VI, Zaragoza, págs. 21-22. 
un primer momento, se pudiera entender que no existe relación entre las distintas culturas ${ }^{22}$.

La interculturalidad, por su parte, hace referencia a la relación e intercambio, y por lo tanto, al enriquecimiento mutuo entre las distintas culturas. Esta conlleva a una comunicación comprensiva entre las distintas culturas que conviven en un mismo espacio, siendo a través de éstas donde se produce el enriquecimiento mutuo y por consiguiente, el reconocimiento y la valoración de cada una de las culturas en un marco de igualdad ${ }^{23}$. «Es el proceso por el cual al menos una cultura es influenciada por otra al establecer una relación en la que hay un proceso en el tiempo caracterizado, por la transferencia de información $»^{24}$.

Establecer categóricamente la interculturalidad implicaría el riesgo de avanzar en la multiculturalidad sin haber alcanzado el reconocimiento y la relación entre distintas culturas, que es lo que implica la multiculturalidad ${ }^{25}$. Es decir, el reconocimiento es entender que una cultura para ser comprendida, tal y como es vivida por aquellos que participan en ella, requiere ser interpretada a partir de sus propios referentes. La interculturalidad postula el reconocimiento incluso desde la incomprensión ${ }^{26}$.

Por ello, la interculturalidad solo tiene sentido si se define como la combinación, en un territorio establecido, de una unidad social y de una pluralidad cultural mediante la comunicación e intercambio entre los distintos actores que utilizan diferentes categorías de expresión análisis e interpretación ${ }^{27}$.

La multiculturalidad es el resultado de una sociedad que reconoce la diversidad o que no reconociéndola ha carecido del poder para homogeneizarse y hacer desaparecer la diferencia. La interacción de los integrantes de diferentes culturas que conviven en un mismo territorio y comparten una realidad semejante, estimula rasgos compartidos

${ }^{22}$ GOIG, Juan (2013). Ob. cit., pág. 1330.

${ }^{23}$ GOIG, Juan (2013). Ob. cit., pág. 1332.

${ }^{24}$ MANSUTTI, Alexander (2008). Dificultades y propuestas sobre el principio de igualdad. Interculturalidad, multiculturalidad, pueblos indígenas y democracia. En Uno y Diverso. Diálogos desde la Diferencia, Primera Edición, Publicaciones Vicerrectorado Académico Universidad de los Andes, Mérida, pág. 112.

${ }^{25}$ GOIG, Juan (2013). Ob. cit., pág. 1335.

26 RODRIGO, Miguel y MEDINA, Pilar (2009). Juventud y Comunicación: el impacto de los medios de comunicación en la transmisión de los valores interculturales, en Juventud y Diálogo entre civilizaciones, Documentos 9, N. ${ }^{\circ} 80$, Injuve, págs. 151-167.

27 TOURAINE, Alan (1995). ¿Qué es una sociedad multiétnica?, en Claves de Razón Práctica, No. 56. 
y la continua adopción y adaptación de estos, puesto que la cultura no es estática, sino que se transforma y adecua constantemente ${ }^{28}$.

Dentro de las dimensiones de la identidad del ser humano, se encuentran sus características culturales y tradicionales practicadas en común con otras personas a lo largo del tiempo. La dignidad del ser humano implica capacidad de elección sobre aspectos esenciales de su vida, uno de los cuales es la identidad, muchos de estos derechos humanos sirven para preservar la identidad de las personas ${ }^{29}$.

En este sentido, un argumento interesante es el que afirma que lo que aportarían al valor individual de cada cultura sus propios integrantes, no es en si el valor de otras culturas consideradas de forma separada, sino el valor de la diversidad, es decir, el reconocimiento de que las culturas sean diferentes y puedan coexistir a nivel social o global $^{30}$.

La diversidad pudiera ser valorada, como una condición esencial para la libertad política o como un motor del desarrollo social. El argumento político se explica puesto que la «coexistencia de varias naciones bajo el mismo Estado es una prueba, y es también la mejor garantía, de su libertad ${ }^{31}$, igualmente se considera que "la libertad provoca la diversidad, y la diversidad preserva la libertad al suministrar los medios de organización» ${ }^{32}$.

El valor de la diversidad radica no solo en la diferencia, sino en que permite y proporciona oportunidades de comunicación entre maneras de ser vividas que son reflectivas, por lo tanto, todo esfuerzo por segregar grupos culturalmente diferenciados y limitar la libertad de asociación y de transferencia de información entre ellos atenta contra la interculturalidad como oportunidad para el desarrollo de la libertad. Una interculturalidad libre se opone tanto a la uniformidad a través de la asimilación como a la diferencia a través de la segregación ${ }^{33}$.

En el caso de los pueblos indígenas, el proceso intercultural se ha desarrollado en un principio como un sistema de agresión sobre las

${ }^{28}$ GOIG, Juan (2013). Ob. cit., pág. 1321.

${ }^{29}$ GOIG, Juan (2013). Ob. cit., pág. 1329.

30 BAUBOCK, Rainer (2009). Justificaciones liberales para los derechos de los grupos étnicos, en Los derechos colectivos. Hacia una efectiva comprensión y protección (María Corredores y María Ávila, editoras), Ministerio de Justicia y Derechos Humanos, Quito, pág. 53.

31 ACTON, J. E. E. D. (1907). Nationality, en The History 01 Freedom and other Essays, London, Macmillan, pág. 290.

32 ACTON, J. E. E. D. (1907). Ob. cit., pág. 289.

33 BAUBOCK, Rainer (2009). Ob. cit., pág. 57. 
cosmovisiones indígenas, siendo el resultado de la violencia ejercida e interiorizada en distintos niveles y ordenes en los que ha sido procesada, tal es el caso en el nivel físico como masacre y genocidio, en el nivel psicológico como memoria indígena arrasada y anulada, en el nivel moral como no reconocimiento de la dignidad y subjetividad indígena, en el nivel cognitivo y cultural como epistemicidio, por ello, para poder permitir la producción, reproducción y desarrollo de las cosmovisiones indígenas es necesario establecer el nivel de las lesiones sufridas por estos grupos para poder construir un proceso multicultural sostenible ${ }^{34}$.

La divergencia que se da entre una diferencia identitaria y otra, es la que deben saber expresar los distintos sistemas normativos a través de mecanismos de interculturalidad que faciliten la mediación y convivencia. La cosmovisión diferente que poseen los pueblos indígenas se expresa también a través de la dimensión colectiva de su identidad, de la naturaleza consuetudinaria de sus estructuras societales y del arraigo en la oralidad de sus códigos culturales. Las cosmovisiones indígenas tienen una compresión de la temporalidad y espacialidad integrada en sus propios procesos culturales, en su forma de concebir los territorios y en la manera de tratar los recursos naturales y la biodiversidad, adquiriendo todas estas comprensiones desde las matrices culturales que las soportan y las recogen ${ }^{35}$.

El ejercicio del derecho a la cultura es contemplado como un conjunto de derechos individuales ejercidos en el seno de la comunidad. Este ejercicio colectivo de los derechos se materializa en el reconocimiento de una cierta autonomía cultural de los individuos que le permite practicar una religión, usar una lengua, libertades políticas o actividades sociales, que, en muchos casos, solo pueden ser ejercidas junto a un número de personas determinado, que pertenecen a los miembros del grupo ${ }^{36}$.

En el caso del Estado venezolano, este se constituye como un Estado con una sociedad multiétnica y pluricultural, conforme a lo establecido en el preámbulo de la Constitución de la República Bolivariana de Venezuela de 1.999, donde en consecuencia «los pueblos indígenas tienen derecho a mantener y desarrollar su identidad étnica y cultura, cosmovisión, valores, espiritualidad y sus lugares sagrados

${ }^{34}$ MARTÍNEZ DE BRINGAS, Asier (2006). Pueblos Indígenas y Derechos Humanos, Universidad de Deusto, Bilbao, pág. 87.

${ }_{35}$ MARTÍNEZ DE BRINGAS, Asier (2006). Ob. cit., pág. 89.

${ }^{36}$ GOIG, Juan (2013). Ob. cit., pág. 1335. 
y de culto» ${ }^{37}$. El derecho a la cultura propia, garantiza la permanencia de la diversidad cultural de las etnias existentes en el país, y es de vital importancia para el respeto de la dignidad de los indígenas. El reconocimiento del derecho a la propia cultura en este caso no trata solo sobre un derecho más en el marco constitucional ${ }^{38}$, sino de un derecho esencial que les ha permitido su reproducción social. La diversidad cultural que representan los pueblos y comunidades indígenas, exige una valoración de la realidad social, de carácter constitutivo del lenguaje en el ámbito de la identidad cultural, en tanto que el principio del pluralismo cultural se sustenta en razón de que la personalidad de los individuos se desarrolla en contextos culturalmente determinados por los valores, las pautas culturales y normativas. El derecho a la propia cultura y la constitucionalización de la diversidad cultural, resulta inherente a la autonomía ética del individuo, se traduce en la aceptación de su idioma, como un bien conectado con el libre desarrollo del individuo, en relación con la cosmovisión de vida que haya interiorizado y las pautas culturales que definen su identidad y personalidad ${ }^{39}$.

Una cultura determinada, posee un horizonte de significación, desde el que se interpreta el mundo, valora, siente y piensa de una manera particular, por lo que conceptos como libertad, castigo, sanción, vida, muerte, conflicto, crimen y justicia, adquieren contenido especifico en razón de la identidad y de las pautas valorativas de cada grupo social, y cuya comprensión solo es posible en un diálogo intercultural ${ }^{40}$, que permita un diálogo sano entre las culturas desde una base no sectaria ni excluyente ${ }^{41}$.

Correspondería al Estado venezolano, el aseguramiento de las libertades de los pueblos indígenas colectiva e individualmente, a fin de que a través del ordenamiento jurídico y de la actuación de los poderes públicos, la cultura de dichos pueblos adquiera el valor que le pertenece en condiciones de igualdad, para que la diversidad cultu-

37 Constitución de la República Bolivariana de Venezuela 1.999. Publicada en Gaceta Oficial de la República de Venezuela Nro. 5453 (Extraordinario), de fecha 24 de marzo de 2000, artículo 121.

${ }^{38}$ FERREIRA, Francisco (2008). Dificultades y propuestas sobre el principio de igualdad. Diversidad cultural y justicia penal: hacia un dialogo intercultural, En Uno y Diverso. Diálogos desde la Diferencia, Primera Edición, Publicaciones Vicerrectorado Académico Universidad de los Andes, Mérida, pág. 157, uno y diverso.

39 Ibidem.

40 Ibidem.

${ }^{41}$ BLÁZQUEZ, Diego (2004). Los derechos de los indígenas desde los clásicos, en Avances en la protección de los derechos de los pueblos indígenas, (Fernando Mariño y J. Daniel Oliva, editores), Instituto Universitario de Estudios Internacionales y Europeos «Francisco de Vitoria. Editorial Dykinson, Madrid, pág. 36. 
ral se traduzca en una concreción real, por lo que el derecho a la cultura propia y el reconocimiento del otro debe ser promovido por el Estado, en atención al respeto y la garantía de la dignidad humana del indígena ${ }^{42}$. Para ello es necesario reconocer las diferencias culturales y de manera concreta las reivindicaciones indígenas en sus pro$\operatorname{cesos}^{43}$.

La Declaración de las Naciones Unidas sobre los Derechos de los Pueblos Indígenas ${ }^{44}$, afirma que los pueblos indígenas son iguales a todos los demás pueblos y reconociendo al mismo tiempo el derecho de todos los pueblos a ser diferentes y a ser respetados como tales, en este sentido el articulo 3 establece: «los pueblos indígenas tienen derecho a la libre determinación. En virtud de ese derecho determinan libremente su condición política y persiguen libremente su desarrollo económico, social y cultural».

En este sentido, la declaración antes citada, garantiza la autonomía de los pueblos indígenas con dicho reconocimiento y la libre determinación en la forma y medida tanto del autogobierno propio como de la vinculación con el Estado. Consiste en la libre determinación de pueblos definidos por cultura ${ }^{45}$. Por ello el proceso multicultural exige que redistribución económica, reconocimiento cultural y participación política, se den simultáneamente.

\section{CONCLUSIÓN}

Es necesario el reconocimiento cultural de los pueblos indígenas con esquemas participativos aptos para construir nuevos modelos económicos interculturales, que determinen nuevas formas distributivas que promuevan procesos de equidad social. Para ello es necesario la participación política en el diseño de nuevas formas de relación entre la sociedad dominante y los pueblos indígenas que permita mitigar las condiciones de exclusión económica y cultural de las que han sido objeto. A tal fin debe procurarse que los pueblos indígenas gocen del derecho a la libre determinación dentro de sus territorios, lo que les permitiría desarrollar ampliamente su condición política, económica, social y cultural.

${ }^{42}$ Ibidem.

${ }^{43}$ MARTÍNEZ DE BRINGAS, Asier (2006). Ob. cit., pág. 98.

44 Declaración de las Naciones Unidas sobre los Derechos de los Pueblos Indígenas, del 13 de septiembre de 2007.

${ }^{45}$ CLAVERO, Bartolomé (2004). Ob. cit., pág. 168. 


\section{REFERENCIAS}

ACTON, J. E. E. D. (1907). Nationality, en The History 01 Freedom and other Essays, London, Macmillan.

BAUBOCK, Rainer (2009). Justificaciones liberales para los derechos de los grupos étnicos, en Los derechos colectivos. Hacia una efectiva comprensión y protección (María Corredores y María Ávila, editoras), Ministerio de Justicia y Derechos Humanos, Quito.

BLÁZQUEZ, Diego (2004). Los derechos de los indígenas desde los clásicos, en Avances en la protección de los derechos de los pueblos indígenas, (Fernando Mariño y J. Daniel Oliva, editores), Instituto Universitario de Estudios Internacionales y Europeos «Francisco de Vitoria. Editorial Dykinson, Madrid.

CLAVERO, Bartolomé (2004). Multiculturalismo Constitucional con perdón, de veras y en frío, en Avances en la protección de los derechos de los pueblos indigenas, (Fernando Mariño y J. Daniel Oliva, editores), Instituto Universitario de Estudios Internacionales y Europeos «Francisco de Vitoria. Editorial Dykinson, Madrid.

COLMENARES, Ricardo (2001). Los derechos de los pueblos indígenas, Editorial Jurídica Venezolana, Caracas.

Comité de Derechos Humanos (1994). Observaciones Generales de los Comités sobre los pueblos indígenas, Observación General No. 23, Los derechos de las Minorías étnicas, religiosas o lingüística, artículo 27 del Pacto.

Constitución de la República Bolivariana de Venezuela 1999. Publicada en Gaceta Oficial de la República de Venezuela Nro. 5453 (Extraordinario), de fecha 24 de marzo de 2000.

DAES, Erica (1997). Protección del patrimonio de los pueblos Indígenas, Informe de la Subcomisión de Prevención de Discriminaciones y Protección a las Minorías, Relatora-Presidenta del Grupo de Trabajo sobre Poblaciones Indígenas.

Declaración de Friburgo sobre los Derechos Culturales, del 07 de mayo de 2007.

Declaración de las Naciones Unidas sobre los Derechos de los Pueblos Indígenas, del 13 de septiembre de 2007.

Declaración de Mataatua, sobre los Derechos de Propiedad Cultural e Intelectual de los Pueblos Indígenas, junio de 1993. 
Declaración de México sobre las Políticas Culturales. Conferencia mundial sobre las políticas culturales México D. F., 26 de julio - 6 de agosto de 1982 .

Declaración Universal de los Derechos del Hombre, Adoptada y Aprobada por la Asamblea General de las Naciones Unidas, del 10 de diciembre de 1948.

DWORKIN, Ronald (1985). A Matter of Principle. Cambridge, Harvard University Press.

FERREIRA, Francisco (2008). Dificultades y propuestas sobre el principio de igualdad. Diversidad cultural y justicia penal: hacia un dialogo intercultural, En Uno y Diverso. Diálogos desde la Diferencia, Primera Edición, Publicaciones Vicerrectorado Académico Universidad de los Andes, Mérida.

GOIG, Juan (2013). Inmigración y Diversidad Cultural. Sobre los «Derechos Culturales de los Inmigrantes», en Teoría y Realidad Constitucional, No. 36.

MANSUTTI, Alexander (2008). Dificultades y propuestas sobre el principio de igualdad. Interculturalidad, multiculturalidad, pueblos indígenas y democracia. En Uno y Diverso. Diálogos desde la Diferencia, Primera Edición, Publicaciones Vicerrectorado Académico Universidad de los Andes, Mérida.

MARTÍNEZ DE BRINGAS, Asier (2006). Pueblos Indígenas y Derechos Humanos, Universidad de Deusto, Bilbao.

MARTÍNEZ DE PISÓN CAVERO, J. (2003). Ciudadanía e inmigración en las sociedades multiculturales, en Ciudadanía e inmigración (Pomed Sanchez, I. y Velasco Caballero, F. Editores), Monografías de la Revista Aragonesa de Administración Pública VI, Zaragoza.

Pacto Internacional de Derechos Civiles y Políticos, del 23 de marzo de 1976.

RODRIGO, Miguel y MEDINA, Pilar (2009). Juventud y Comunicación: el impacto de los medios de comunicación en la transmisión de los valores interculturales, en Juventud y Diálogo entre civilizaciones, Documentos 9, No. 80, Injuve.

RUIZ, Oswaldo (2004). El derecho a la identidad cultural de los pueblos indígenas y la minorías nacionales: Una mirada desde el sistema interamericano, en SUR - Revista Internacional de Derechos Humanos, No. 5, Año 3.

TOURAINE, Alan (1995). ¿Qué es una sociedad multiétnica?, en Claves de Razón Práctica, No. 56. 
